\title{
Gluon polarisation in the nucleon from COMPASS
}

\section{Krzysztof Kurek ${ }^{* \dagger}$}

On behalf of COMPASS Collaboration.

Andrzej Soltan Institute for Nuclear Studies, Hoza 69, 00-681 Warsaw, Poland

E-mail: kurek@fuw.edu.pl

New results on the longitudinal inclusive asymmetry $A_{1}^{d}$ and the spin-dependent structure function $g_{1}^{d}$ is the range $0.002(\mathrm{GeV} / \mathrm{c})^{2}<Q^{2}<100(\mathrm{GeV} / \mathrm{c})^{2}$ and $0.00004<x<0.7$ and new measurements of the gluon polarization $\Delta G / G$ via the open charm channel and based on the helicity asymmetry of large transverse-momentum hadrons in the final state are presented. The data have been collected in the years 2002-2004 by the COMPASS experiment at CERN using a $160 \mathrm{GeV} / \mathrm{c}$ polarized muon beam scattered off a polarized ${ }^{6} \mathrm{LiD}$ target. The longitudinal asymmetry results in the low $x(0.00004<x<0.03)$ and low $Q^{2}\left(0.002(\mathrm{GeV} / \mathrm{c})^{2}<Q^{2}<1(\mathrm{GeV} / \mathrm{c})^{2}\right)$ region are consistent with zero and the precision increased ten times compared to the results of the SMC, so far the only experiment which has measured the low $x$ and low $Q^{2}$ domain. The results on the spin-dependent structure function $g_{1}^{d}$ measured in DIS region $\left(Q^{2}>1(\mathrm{GeV} / \mathrm{c})^{2}\right)$ are in agreement with those from previous experiments, improve considerable the statistical accuracy for $0.004<x<0.03$ region and have been included in new COMPASS NLO QCD analysis of the world data. The new preliminary result for $\Delta G / G$ from the charm channel is $-0.57 \pm 0.41$ (stat.) at $x_{G} \simeq 0.15$ and scale $\mu^{2} \simeq 13(\mathrm{GeV} / \mathrm{c})^{2}$.

The gluon polarization from high- $p_{T}$ hadron pairs is $\Delta G / G=0.016 \pm 0.058$ (stat. $) \pm 0.055$ (syst.) at $x_{G} \simeq 0.085_{-0.035}^{+0.07}\left(Q^{2}<1(\mathrm{GeV} / \mathrm{c})^{2}\right.$ and $\left.\mu^{2} \simeq 3(\mathrm{GeV} / \mathrm{c})^{2}\right)$.

DIFFRACTION 2006 - International Workshop on Diffraction in High-Energy Physics

September 5-10 2006

Adamantas, Milos island, Greece

${ }^{*}$ Speaker.

${ }^{\dagger}$ This work was partially supported by SPUB 621/E-78/SPB CERN/P-03 


\section{Introduction.}

The EMC spin asymmetry measurement $[1,2]$ and the naive interpretation of the results following from the Ellis-Jaffe sum rule [3] have introduced the so-called "spin crisis": quarks carry a very small fraction of the nucleon's helicity. The next experiments at CERN, DESY and SLAC confirmed that quarks are only responsible for roughly $1 / 3$ of nucleon's helicity. The quark helicity distributions $\Delta q_{i}\left(x, Q^{2}\right)$ are related to a vector-axial quark current which is not conserved due to the Adler-Bell-Jackiw anomaly. This fact allows to explain the spin crisis by changing the interpretation of the measurement: instead of quark spin contents $\Delta \Sigma=\int_{0}^{1} \sum_{i=1}^{n_{f}} \Delta q_{i}\left(x, Q^{2}\right) d x$ the combination $\Delta \Sigma-\left(3 \alpha_{s}\right) /(2 \pi) \Delta G$ is measured, where $\Delta G$ is a gluon polarization inside the nucleon. The spin crisis and the violation of the Ellis-Jaffe sum rule can be then avoided if $\Delta G$ is large enough. This interpretation was a "driving force" in preparation a series of new polarized DIS type experiments related to direct measurement of $\Delta G$ : HERMES in DESY, SMC and COMPASS at CERN, STAR and PHOENIX at RHIC.

To complete the picture, beside the quark's helicity $\Delta \Sigma$, and the gluon polarization $\Delta G$ also an orbital angular momentum of quarks and gluons can build the nucleon spin structure. In this paper I will present new results of the longitudinal inclusive asymmetry $A_{1}^{d}$ and the spin-dependent structure function $g_{1}^{d}$ and new results for a direct measurement of gluon polarization $\Delta G / G$ obtained by COMPASS collaboration after analyzing the data sets collected in years 2002-2004. The experiment is using a $160 \mathrm{GeV} / \mathrm{c}$ polarized muon beam from the SPS at CERN scattered off a polarized ${ }^{6} \mathrm{LiD}$ target (for more details see [4]). The paper is organized as follows. In Section 2 the longitudinal inclusive asymmetry $A_{1}^{d}$ and the $g_{1}^{d}$ structure function for small $x$ and small $Q^{2}$ domain are presented. The $A_{1}^{d}$ asymmetry and the $g_{1}^{d}$ structure function results for the DIS region $\left(Q^{2}>1\right.$ $\left.(\mathrm{GeV} / \mathrm{c})^{2}\right)$ and the results of the perturbative QCD analysis of the world data are presented in Section 3. The gluon polarization measurement results and conclusions are presented in Section 4, 5 and 6 , respectively.

\section{The longitudinal helicity asymmetry $A_{1}^{d}$ for small $x$ and small $Q^{2}$ domain.}

The cross-section longitudinal helicity asymmetry:

$$
A_{L L}^{d}=\frac{\sigma^{\leftrightarrows}-\sigma^{\leftrightarrows}}{\sigma^{\leftrightarrows}+\sigma^{\leftrightarrows}}
$$

can be decomposed into the virtual photon-deuteron asymmetry $A_{1}^{d}$ and $A_{2}^{d}$ in the following way:

$$
A_{L L}^{d}=D\left(A_{1}^{d}+\eta A_{2}^{d}\right) \simeq D A_{1}^{d}
$$

where photon depolarization factor $D$ (as well as $\eta$ ), depends on the event kinematics. Arrows correspond to relative orientation of the incoming muon and the target deuteron helicities and all factors which contain $A_{2}^{d}$ have been neglected since they are very small. The spin-dependent structure function $g_{1}^{d}$ is related to the asymmetry $A_{1}^{d}$ as follows:

$$
g_{1}^{d} \simeq \frac{F_{2}^{d}}{2 x(1+R)} A_{1}^{d}
$$




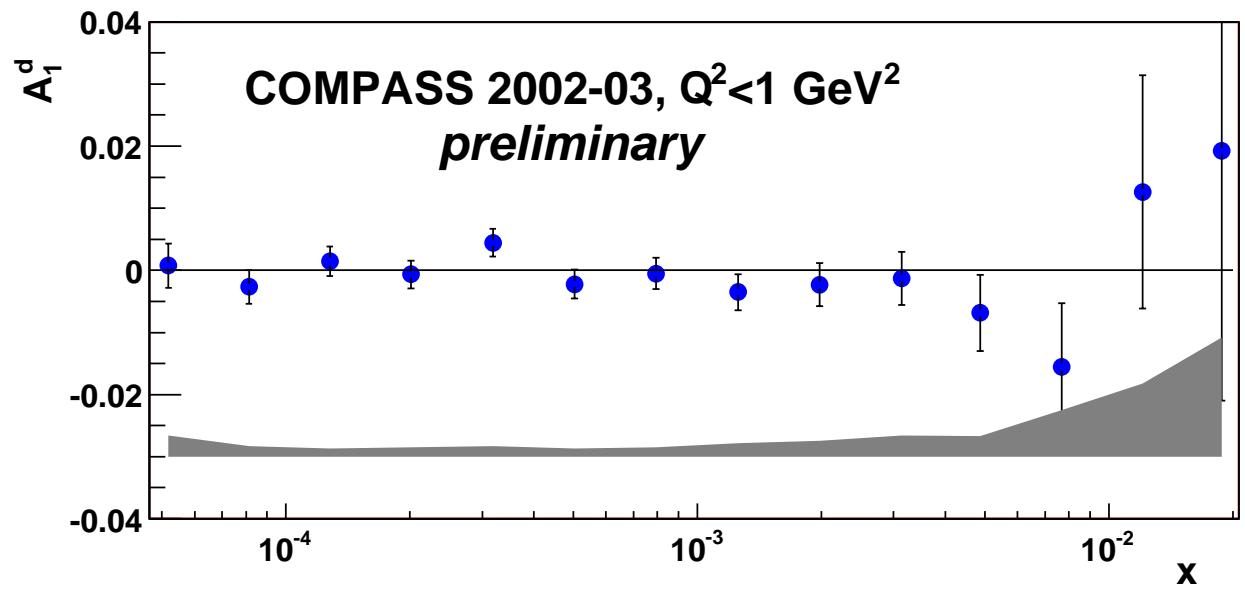

Figure 1: The COMPASS results of the $A_{1}^{d}$ in the low $x$ and low $Q^{2}$ region.

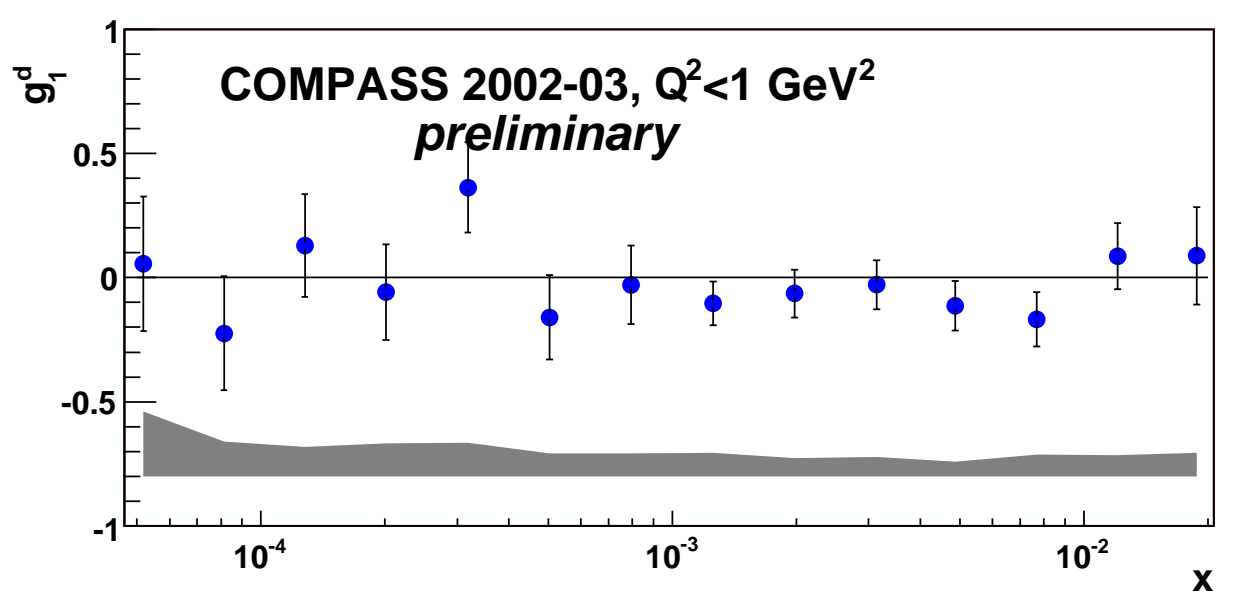

Figure 2: The COMPASS results of the $g_{1}^{d}$ in the low $x$ and low $Q^{2}$ region.

where $F_{2}^{d}$ and $R$ are unpolarized (spin independent) structure functions.

The asymmetry and the $g_{1}$ structure function have been calculated for events with small $Q^{2}\left(Q^{2}<1\right.$ $\left.(\mathrm{GeV} / \mathrm{c})^{2}\right)$ and small $x(x>0.00004)$. The presented data come from the years 2002 and 2003 . The final sample used in the analysis contains 300 milion events.

The values of $F_{2}$ for $x>0.0009$ and $Q^{2}>0.2(\mathrm{GeV} / \mathrm{c})^{2}$ have been taken from [5] and from [6] in the rest of the phase space. $R$ comes from [7] for $Q^{2}>0.5(\mathrm{GeV} / \mathrm{c})^{2}$. For lower $Q^{2} R$ is proportional to $Q^{2}$ at the photoproduction limit. The results for the asymmetry $A_{1}^{d}$ as a function of $x$ are presented in Figure 1. Figure 2 shows the results on the $g_{1}^{d}$ structure function. The shadowed bands indicate the systematics errors and the error bars with the data points mark statistical ones. The results are consistent with zero in the considered $x$ range.

Comparison with the SMC results [8] for $Q^{2}<1(\mathrm{GeV} / \mathrm{c})^{2}$ is shown in Figure 3. The statistical 


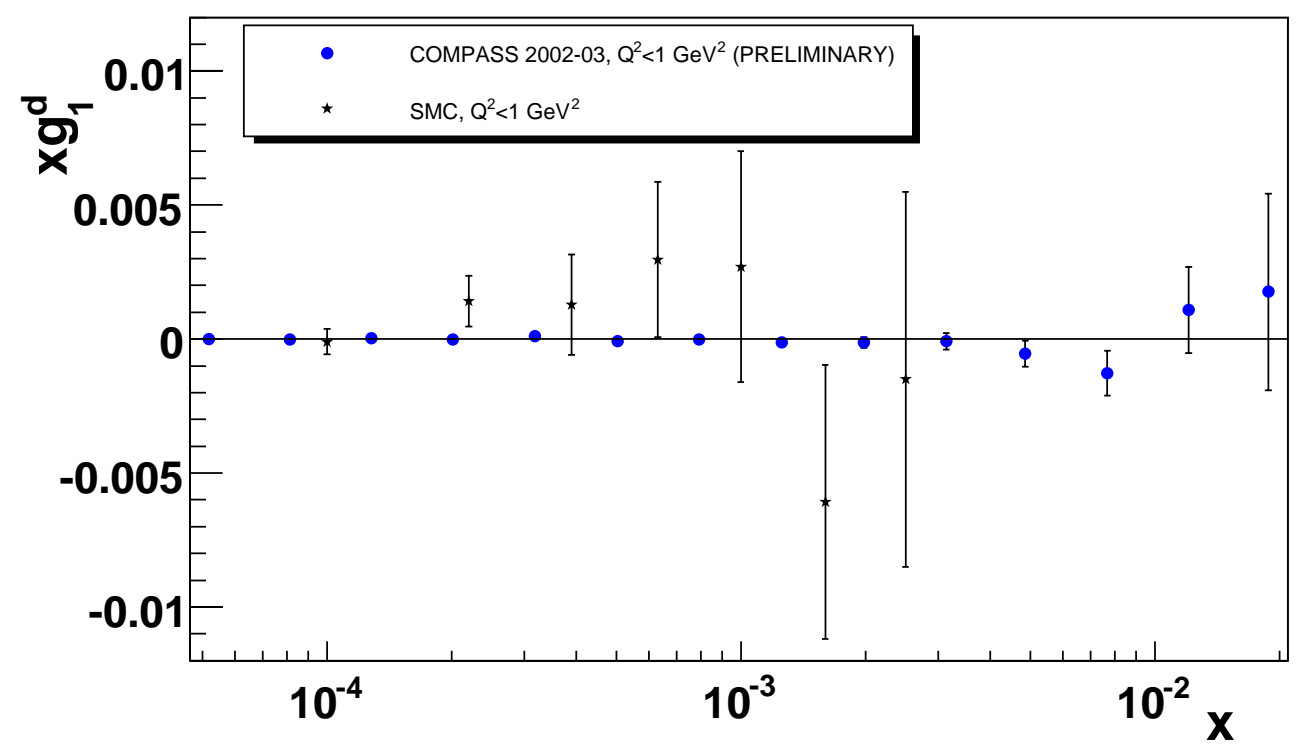

Figure 3: The comparison of the $g_{1}^{d}$ results with the SMC results for the low $Q^{2}$ region. Only statistical errors are shown with the data points.

precision of $A_{1}^{d}$ and $g_{1}^{d}$ in the COMPASS is ten times higher than in the SMC one. The SMC and the COMPASS results are consistent in the overlap region.

\section{3. $A_{1}^{d}$ asymmetry and $g_{1}^{d}$ structure function in for high $Q^{2}$. QCD analysis.}

In the Figure 4 the results on the $A_{1}^{d}$ asymmetry for $Q^{2}>1(\mathrm{GeV} / \mathrm{c})^{2}$ (DIS domain) as a function of $x$ are shown as measured in COMPASS and superposed to results of previous experiments at CERN [5], DESY [9] and SLAC [10]. Again, small terms related to $A_{2}^{d}$ have been neglected. The data were collected during the years 2002-2004. The resulting sample consists of 89 milion events. The asymmetry results from 2002-2003 data have been published in [11]. Full data sample results are being published in [12].

The asymmetry is consistent with zero for $x<0.03$. The spin-dependent structure function $g_{1}^{d}$ has been calculated with $F_{2}^{d}$ parametrization of [5] and the $R$ parametrization has been taken from [7].

A new NLO QCD fit of all $g_{1}$ data at $Q^{2}>1(\mathrm{GeV} / \mathrm{c})^{2}$ from deuteron [5, 9, 10, 13] (including new COMPASS data), proton [2, 5, 9, 10, 14] and ${ }^{3} \mathrm{He}$ [15] targets has been performed. In total 230 data points have been used. The NLO fits have been performed in $\overline{M S}$ scheme with input parametrizations at $Q^{2}=3(\mathrm{GeV} / \mathrm{c})^{2}$ of the quark singlet spin distribution $\Delta \Sigma(x)$, the non-singlet distributions $\Delta q_{3}(x)$ and $\Delta q_{8}(x)$ and the gluon distribution function $\Delta G(x)$ in the form:

$$
\Delta F_{k}=\eta_{k} \frac{x^{\alpha_{k}}(1-x)^{\beta_{k}}\left(1+\gamma_{k} x\right)}{\int_{0}^{1} x^{\alpha_{k}}(1-x)^{\beta_{k}}\left(1+\gamma_{k} x\right) d x} .
$$



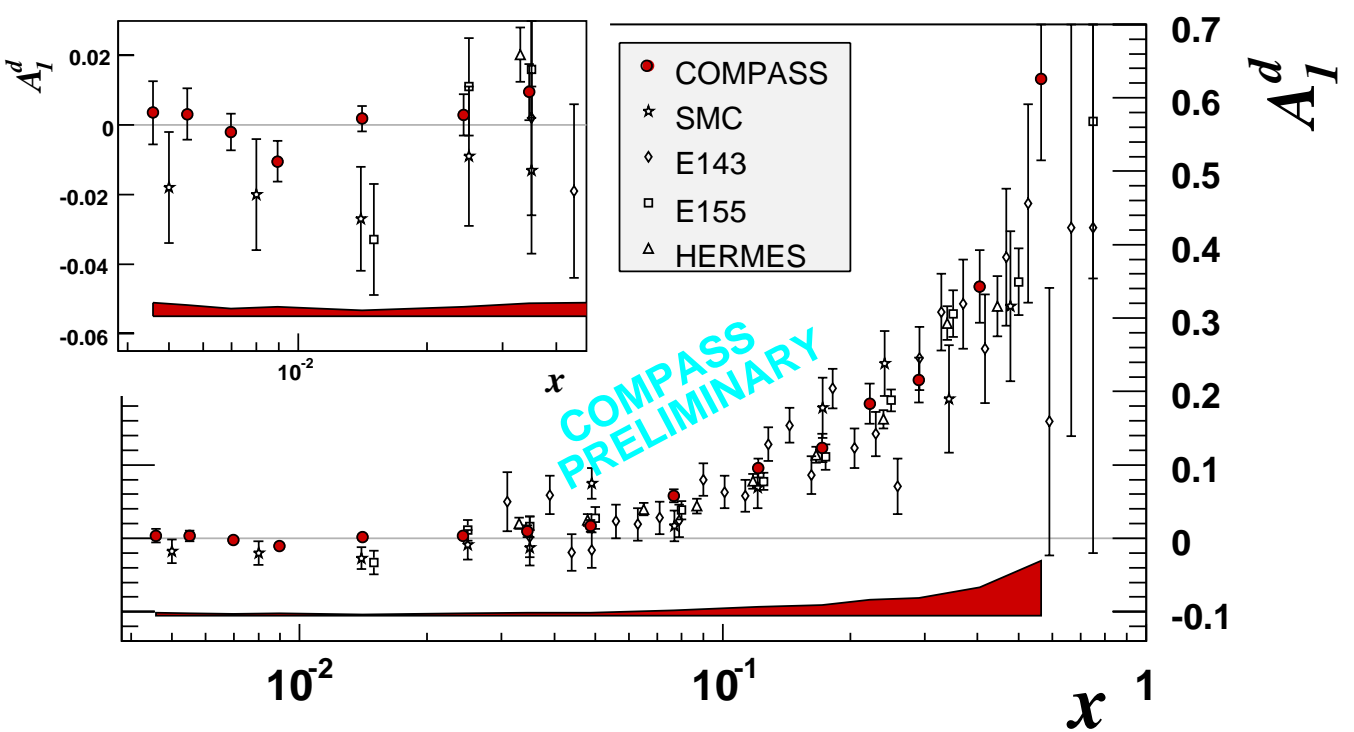

Figure 4: The asymmetry $A_{1}^{d}(x)$ for $Q^{2}>1(\mathrm{GeV} / \mathrm{c})^{2}$. Only statistical errors are shown with a data points. The COMPASS systematic errors are marked by shadowed areas.

The distributions have been evolved according to the DGLAP equations. The moments $\eta_{k}$ for the non-singlet distributions $\Delta q_{3}(x)$ and $\Delta q_{8}(x)$ have been fixed by the baryon decay constants $(F+D)$ and $(3 F-D)$ respectively [16], assuming $\mathrm{SU}(3)_{f}$ symmetry. The linear term $\gamma x$ has been used only for singlet distribution. $\beta_{G}$ has been fixed because it is poorly constrained by the data. Finally 10 parameters in the input distributions have been fitted. In order to keep the parameters in the physical range, the polarized strange sea and gluon distributions have been required to satisfy the so-called positivity condition: $|\Delta s(x)| \leq s(x)$ and $|\Delta G(x)| \leq G(x)$ at all $Q^{2}$ values. The unpolarized distributions in this test have been taken from the MRST parametrization [17]. The fit has been performed with two different programs [18] which give consistent values of the fitted parameters and similar $\chi^{2}$-probabilities. Each program yields two solutions, one with $\Delta G$ positive, the other with $\Delta G$ negative. The $g_{1}^{d}$ structure function results evolved to $Q^{2}=3(\mathrm{GeV} / \mathrm{c})^{2}$ and the results of the fit are shown in Figure 5. Previous fits of the $g_{1}$ structure function, not including the COMPASS data, found positive $\Delta G$ and the fitted $g_{1}^{d}(x)$ getting negative for $x \leq 0.025$ at $Q^{2}=3(\mathrm{GeV} / \mathrm{c})^{2}$. The new COMPASS data do not show any evidence for a decrease of the structure function at small $x$. The COMPASS values of $g_{1}^{N}$ defined as $\left(g_{1}^{p}+g_{1}^{n}\right) / 2$ and expressed by $g_{1}^{d}$ :

$$
g_{1}^{N}\left(x, Q^{2}\right)=\frac{g_{1}^{d}\left(x, Q^{2}\right)}{\left(1-3 / 2 \omega_{D}\right)},
$$

where $\omega_{D}=0.05 \pm 0.01$ is a correction for the D-wave state of the deuteron [19], evolved to $Q^{2}=3$ $(\mathrm{GeV} / \mathrm{c})^{2}$ are shown in Figure 6 together with previous published fits and the new COMPASS fits. The more details concerning NLO QCD COMPASS fits and the evaluation of the first moment of the structure function $g_{1}^{d}$ can be found in [12].

The disagreement of the COMPASS data with previous QCD fits for the small $x$ region is seen from the comparison in Figure 6 and the existing QCD parametrizations need to be revised. 


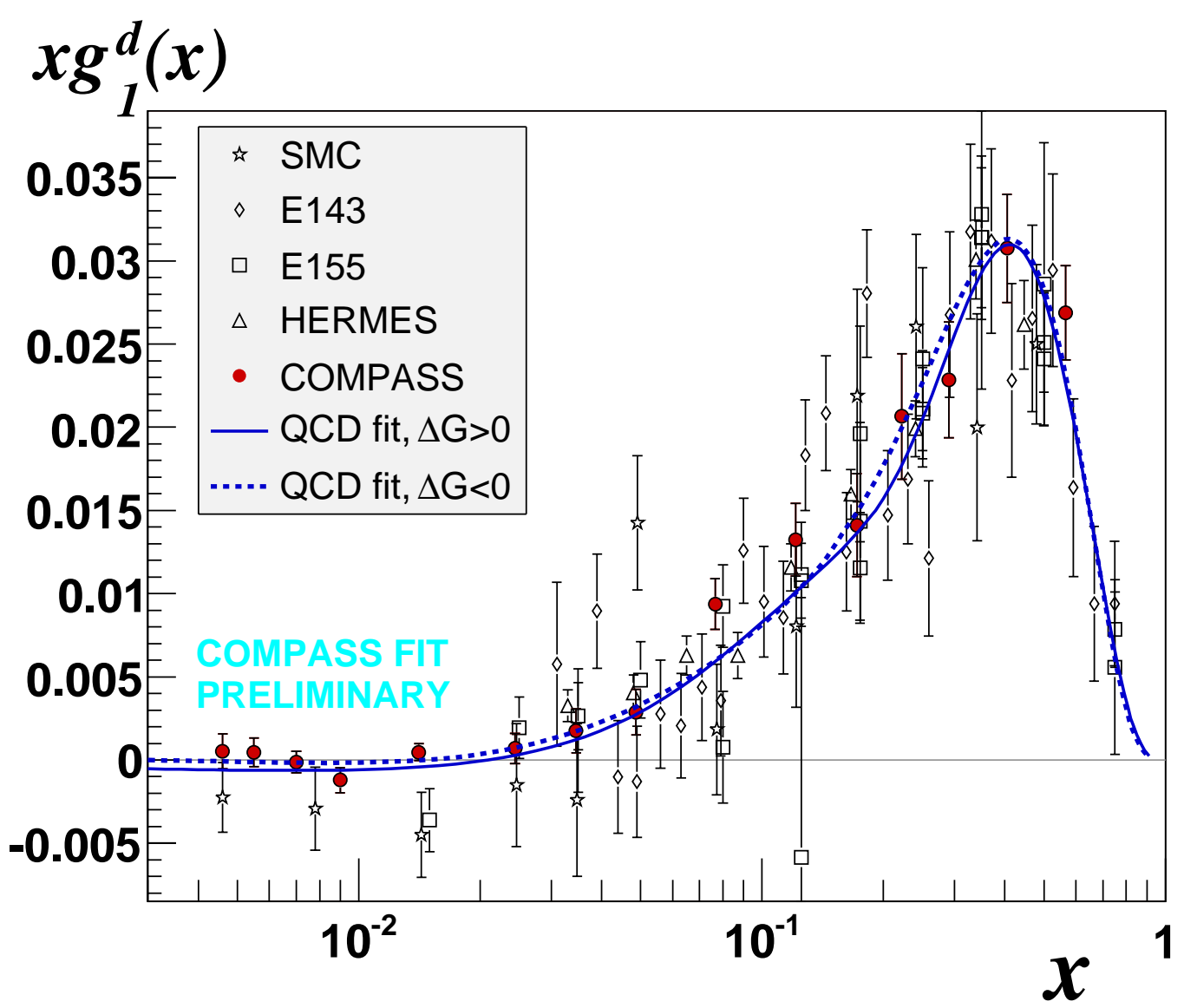

Figure 5: Measured values of $x g_{1}^{d}(x)$ evolved to $Q^{2}=3(\mathrm{GeV} / \mathrm{c})^{2}$. Only statistical errors are shown with data points. The curves show the preliminary results of QCD fits with $\Delta G>0$ and $\Delta G<0$. The updated results of the fits are being published in [12].

\section{Direct measurement of gluon polarization. $\Delta G / G$ from open charm channel.}

In the LO QCD approximation the only subprocess which probes gluons inside nucleon is Photon-Gluon Fusion (PGF). There are two ways allowing direct access to gluon polarization via the PGF subprocess available in the COMPASS experiment: the open charm channel where the events with reconstructed $D^{0}$ mesons are used and the production of two hadrons with relatively high- $p_{T}$ in the final state. The open charm channel guarantees no physical background because the PGF subprocess is the only possible mechanism for charm quark pair production in LO QCD approximation (NLO corrections, the so-called "intrinsic" charm mechanism as well as resolved photon contribution are neglected in this analysis). Therefore the estimation of the gluon polarization in this case is much less Monte-Carlo (MC) dependent than in the two high- $p_{T}$ hadrons method, where the complicated background requires very good MC description of the data. On the other hand the statistical precision in high- $p_{T}$ hadrons method is much higher than in the open charm channel. In this Section the new COMPASS results of $\Delta G / G$ from the open charm channel is discussed. 


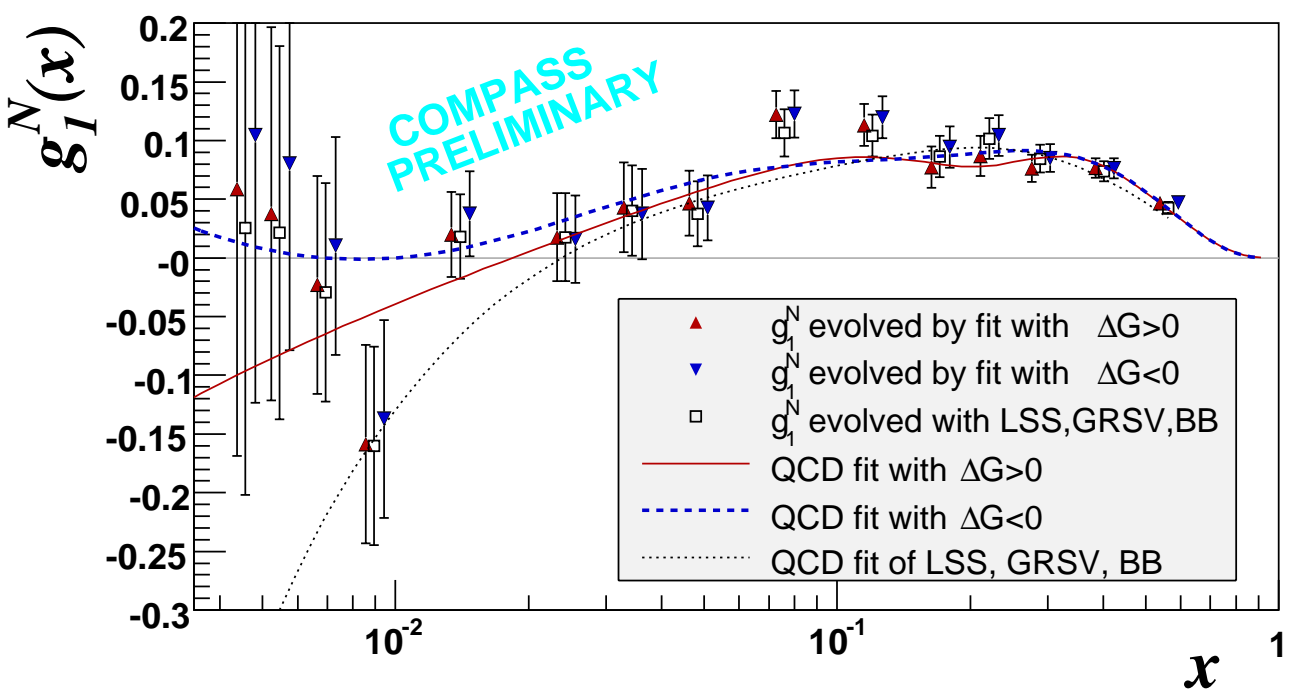

Figure 6: The COMPASS values of $g_{1}^{d}$ evolved to $Q^{2}=3(\mathrm{GeV} / \mathrm{c})^{2}$. In addition to the COMPASS preliminary fits the curve obtained from three published parametrizations [20] is shown. These parametrizations lead almost to the same values of $g_{1}^{N}$ and have been averaged. The data points evolved with different fits are shifted on $x$ with respect to each other. Only statistical errors are shown.

For events with charm quark production a helicity asymmetry is measured. Charm quarks are tagged by measuring $D$ mesons in one of the two channels: the $D^{0}$ meson decaying into the "golden" channel i.e. to a kaon and a pion, and $D^{*}$ decaying into soft pion and a $D^{0}$ with subsequent decay (so-called $D^{*}$-tagged events). For particle identification the RICH detector was used. The gluon polarization $\Delta G / G$ is related to the measured helicity asymmetry $A_{L L}\left(A_{L L} \equiv A_{L L}^{d}\right.$ in this and next Sections) as follows:

$$
A_{L L}=\frac{S}{S+B} a_{L L} \frac{\Delta G}{G}
$$

where $S$ and $B$ denote signal and combinatorial background, respectively, and $a_{L L}$ is the analyzing power - the ratio of spin-dependent and spin-independent cross sections in the PGF process and is given as a function of photon as well as gluon kinematics. The photon kinematics can be fully reconstructed based on the incoming and scattered muons but the gluon part cannot be reconstructed because only one charmed meson is measured. From MC studies it was shown that the knowledge of the kinematics of only one charmed meson can be used to reconstruct the analyzing power approximately. The parametrization of $a_{L L}$ was found by a neural network trained using MC sample generated by the AROMA generator and reconstructed as for real data. Figure 7 shows the value of $\Delta G / G$ as a function of year of data taking separately for $D^{*}$-tagged and un-tagged events. Combining the data from 2002-2004 we obtain the following preliminary COMPASS result for the gluon polarization from the open charm channels: $\Delta G / G=-0.57 \pm 0.41$ (stat.) at $x_{G} \simeq 0.15$ and with the scale $\mu^{2} \simeq 13(\mathrm{GeV} / \mathrm{c})^{2}$. The systematic error studies are ongoing. We expect the systematical uncertainty of the result to be smaller than statistical error. 


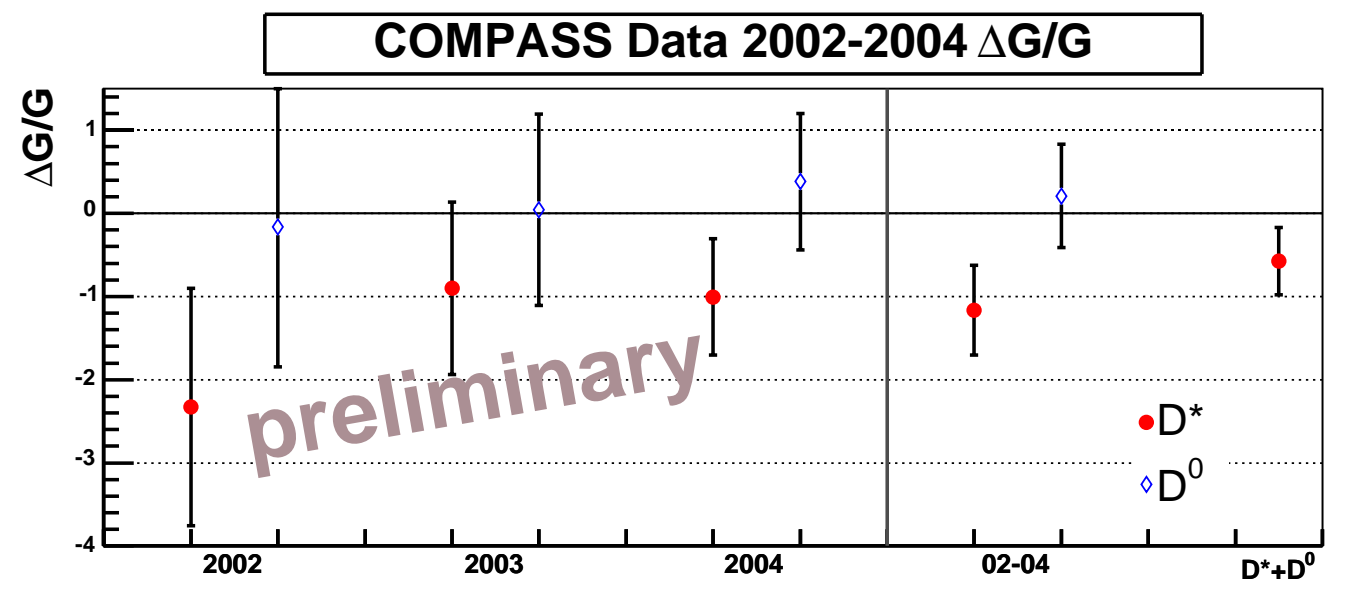

Figure 7: The value of $\Delta G / G$ obtained from measured helicity asymmetries for charm events.

\section{5. $\Delta G / G$ from two high- $p_{T}$ hadrons.}

Two parallel high- $p_{T}$ hadrons analyses are going on: the so-called quasi-real photoproduction $\left(Q^{2}<1(\mathrm{GeV} / \mathrm{c})^{2}\right)$ of high- $p_{T}$ hadron pairs and the high- $p_{T}$ hadrons analysis with high $Q^{2}\left(Q^{2}>1\right.$ $\left.(\mathrm{GeV} / \mathrm{c})^{2}\right)$. The reason for performing the analysis in the two kinematical regions separately is that in the two cases different background processes are contributing. Corrections for this background have to be taken from MC simulations and therefore very good agreement between data and MC is required. The helicity asymmetry for two high- $p_{T}$ hadrons is expressed as follows:

$$
A_{L L}=R_{P G F} a_{L L} \frac{\Delta G}{G}+A_{B k g},
$$

where again $a_{L L}$ is the analyzing power for PGF subprocess, $R_{P G F}$ is a fraction of PGF processes (taken from $\mathrm{MC}$ ) and $A_{B k g}$ denotes the asymmetry from different background processes which contribute to the observed two hadron final state. In the low $Q^{2}$ sample the complicated background, including resolved photon contribution was simulated with the PYTHIA MC generator. An important contribution to the systematic error is related to the unknown polarized distribution functions in the photon (two scenarios: plus or minus maximal polarization were taken into account). The detailed procedure, data/MC comparison and results for $\Delta G / G$ obtained for 20022003 data have been recently published [21]. The preliminary result - including the 2004 data is: $\Delta G / G=0.016 \pm 0.058$ (stat. $) \pm 0.055$ (syst. $)$ at $x_{G} \simeq 0.085_{-0.035}^{+0.07}$ and scale $\mu^{2} \simeq 3(\mathrm{GeV} / \mathrm{c})^{2}$. The comparison of all COMPASS $\Delta G / G$ results and the results from SMC [22] and HERMES [23] experiments is presented in Figure 8 together with the $\Delta G(x)$ results from new COMPASS NLO QCD fits.

A new result for the high $Q^{2}$ region - including the 2004 data set is expected soon. In contrast to low $Q^{2}$ the physical background is dominated by the leading and the QCD-Compton process. The LEPTO MC generator is used in this analysis. Due to the fact that large $Q^{2}$ guarantees a perturbative scale the $p_{T}$ cuts can be released increasing statistics. A neural network approach similar to the one used in the SMC analysis [22] is now tested. We expect the reduction of the statistical 


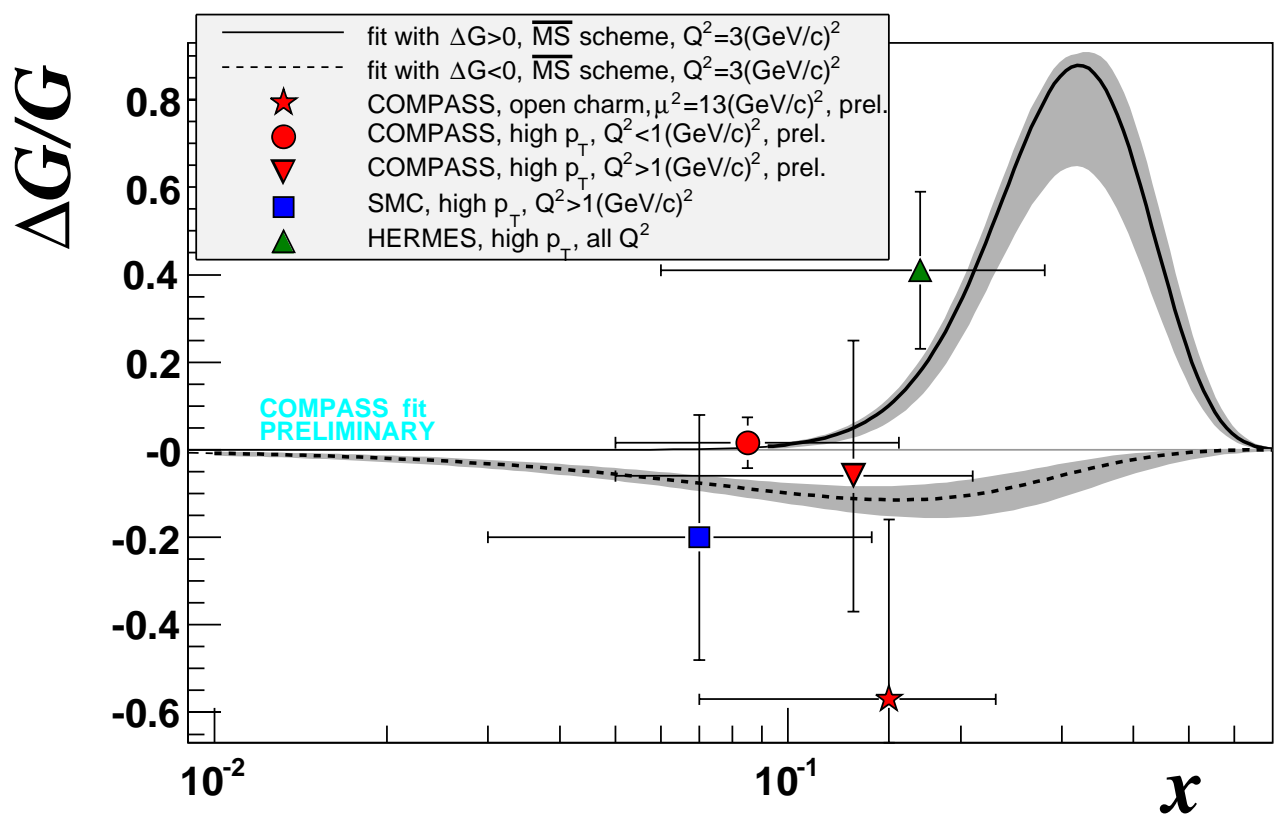

Figure 8: Comparison of the $\Delta G / G$ measurement from COMPASS, SMC and HERMES and the distribution of the gluon polarization $\Delta G(x) / G(x)$ at $Q^{2}=3(\mathrm{GeV} / \mathrm{c})^{2}$ for the preliminary fits with $\Delta G>0$ and $\Delta G<0$ obtained with the fitting program of COMPASS. The error bands correspond to the statistical error on $\Delta G(x)$ at given $x$.

error by the factor up to 4 including 2004 data.

At the end it is worth to note that NLO corrections are partially taken into account by using parton showers in MC generators and string type fragmentation functions. Although model (MC) dependent - the obtained result for low $Q^{2}$ events is the most precise estimation of the $\Delta G / G$ from directly measured helicity asymmetries.

\section{Conclusions.}

The new results of the longitudinal inclusive helicity asymmetry $A_{1}^{d}$ measured in the range $0.002(\mathrm{GeV} / \mathrm{c})^{2}<Q^{2}<100(\mathrm{GeV} / \mathrm{c})^{2}$ have been presented. The asymmetry for small $Q^{2}$ domain corresponds to very small $x$ : $0.00004<x<0.03$ and is consistent with zero. The DIS events $\left(Q^{2}>1(\mathrm{GeV} / \mathrm{c})^{2}\right)$ cover $x$ region from 0.004 up to 0.7 . The COMPASS results are in agreement with those from previous experiments and improve considerably the statistical accuracy in the small $x$ region. For DIS events the results of new NLO QCD fits have been presented and compared with the previous published world data fits. The COMPASS small $x$ data indicate disagreement with the previous published fits for small $x$.

The new measurements of the gluon polarization obtained from the COMPASS experiment have been presented. The model-independent direct measurement based on the open charm channel and the most precise but model (MC) dependent result from two high- $p_{T}$ hadron pair analysis indicate that a small $\Delta G$ is preferred. The Ellis-Jaffe sum rule seems to be violated if a large $\Delta G$ is excluded. 
The small value of $\Delta G$ indicates important role of angular orbital momentum in the nucleon spin decomposition in the frame of the parton model and perturbative QCD.

\section{References}

[1] J. Ashman et al., Phys. Lett. B206 (1988) 364.

[2] J. Ashman et al., Nucl. Phys. B328 (1989) 1.

[3] J. Ellis and R. L. Jaffe, Phys. Rev. D9 (1974) 1444, Phys. Rev. D10 (1974) 1669.

[4] G. K. Mallot, for the COMPASS Collaboration, Nucl. Instrum. Meth. A518 (2004) 121.

[5] SMC, B. Adeva et al., Phys. Rev. D58 (1998) 112001; Erratum ibid. $D 62079902$.

[6] B. Badełek and J. Kwieciński, Phys. Lett. B295 (1992) 263.

[7] E143, K. Abe et al., Phys. Lett. B452 (1999) 194.

[8] SMC, B. Adeva et al., Phys. Rev. D60 (1999) 072004.

[9] HERMES, A. Airapetian et al., Phys. Rev. D75 (2005) 012003.

[10] E143, K. Abe et al., Phys. Rev. D58 (1998) 112003.

[11] COMPASS, E. S. Ageev et al., Phys. Lett. B612 (2005) 154.

[12] COMPASS, E. S. Ageev et al., hep-ex/0609038, accepted in Phys. Lett. B.

[13] E155, P. L. Anthony et al., Phys. Lett. B463 (1999) 339.

[14] E155, P. L. Anthony et al., Phys. Lett. B493 (2000) 19.

[15] E142, P. L. Anthony et al., Phys. Rev. D54 (1996) 6620; E154, K. Abe et al., Phys. Rev. Lett. 79 (1997) 26; JLAB/Hall A, A .X. Zheng et al., Phys. Rev. Lett. 92 (2004) 012004; HERMES, K. Ackerstaff et al., Phys. Lett. B404 (1997) 383.

[16] Y. Goto et al., Phys. Rev. D62 (2000) 034017.

[17] A. D. Martin et al., Eur. Phys. J. C4 (1998) 463.

[18] SMC, B. Adeva et al., Phys. Rev. D58 (1998) 112002.; A. N. Sissakian, O. Yu. Shevchenko and O. K. Ivanov, Phys. Rev. D70 (2004) 074032.

[19] R. Machleid et al., Phys. Rep. 149 (1987) 1.

[20] The Durham HEP Database, http://durpdg.dur.ac.uk/HEPDATA/pdf.html.

[21] COMPASS, E. S. Ageev et al., Phys. Lett. B633 (2006) 25.

[22] SMC, B. Adeva et al., Phys. Rev. D70 (2004) 012002.

[23] HERMES, A. Airapetian et al., Phys. Rev. Lett. 84 (2000) 2584. 